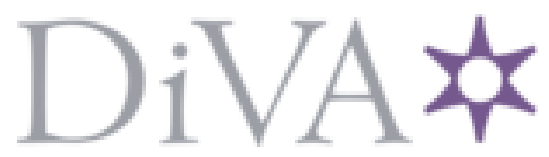

http://www.diva-portal.org

This is the published version of a paper presented at SIGGRAPH Asia 2013.

Citation for the original published paper:

Lu, Z., ur Réhman, S. (2013)

Touch-less interaction smartphone on go!.

In: Proceeding of SIGGRAPH Asia 2013 ACM New York, NY, USA

http://dx.doi.org/10.1145/2542302.2542336

N.B. When citing this work, cite the original published paper.

Permanent link to this version:

http://urn.kb.se/resolve?urn=urn:nbn:se:umu:diva-83879 


\title{
Touch-less Interaction Smartphone On Go!
}

\author{
Zhihan LU*, Shafiq ur Réhman \\ Shenzhen Institutes of Advanced Technology, Chinese Academy of Science, 518055, China \\ i2lab, Department of Applied Physics and Electronics, Umea University, Umea, Sweden
}

\section{Abstract}

A smartphone touch-less interaction based on mixed hardware and software is proposed in this work. The software application renders circle menu application graphics and status information using smart phone's screen, audio. Augmented reality image rendering technology is employed for a convenient finger-phone interaction. The users interact with the application using finger gesture motion behind the camera, which trigger the interaction event and generate activity sequences for interactive buffers. The combination of Contour based Template Matching (CTM) and Tracking-Learning-Detection (TLD) provides a core support for hand-gesture interaction by accurately detecting and tracking the hand gesture.

\section{Introduction}

Touch-less interaction extends the operation space for interacting with mobiles and helps mitigate the problems of using mobile phone while user's hands are not available, for instance, their hands are dirty, or they are wearing gloves in winter, or they are driving and holding the steering wheel. In addition, touch-less interactions support more gestures in more natural patterns [1]. We present a touch-less interaction approach for smart phones where user mounts smart phone over wrist and performs 'in air' interaction gestures.

\section{Our approach}

Our approach uses fixing the smartphone on the forearm by a lowcost flexible hardware and tracking the finger motion (behind the screen) to simulate the touch event by a novel algorithm. Our algorithm uses dynamic programming based CTM algorithm [2] to localize the finger-contour and employs TLD [3] framework to strengthen the tracking process in every frame. TLD algorithm combines elements from tracking, learning and detection in the 2D image space to make it a long-term tracker. The TLD tracker uses a tracking strategy of the overlapping blocks, and tracks every block by Lucas-Kanade optical flow method. CTM approach is an indispensable step for interactive hand (two fingers outstretched) detection in the beginning. CTM gives TLD a region of interest (ROI) for initialization.

Our algorithm execution time is real time and accurate even when the hand gesture is moving fast and in image blur cases. It's worth mentioning that the method can detect all kinds of the handgesture used in our application, as long as the correct hand texture is detected in the first frame.

To improve the efficiency of the proposed algorithm, the size of first frame is scaled down to $25 \%$ and then CTM is applied. From the second frame the image is scaled to $12.5 \%$ and then TLD tracking is employed. The 'hand target test' results indicate both efficiency and success rate of the tracking is close to $100 \%$.

\section{Results}

To verify the accuracy of our algorithm we have tested it on our dataset; which contained 120 mobile video sequences (more than
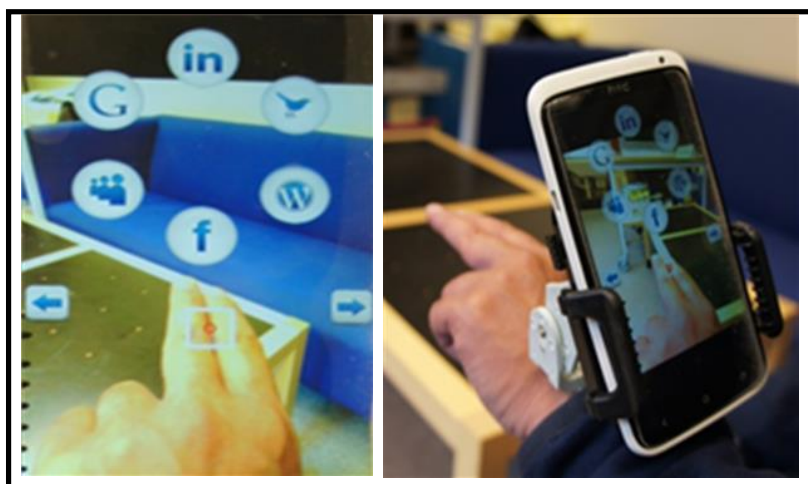

Figure 1. [From left to right] User interaction space shown on smart phone's screen augmented with virtual objects.

Complete Application Scenario

1000 frames each) of single hand-gesture for various sizes. Our dataset contained video-sequences from clustered office as well as outdoor lighting conditions. The proposed algorithm successfully located and tracked the hand-gesture in almost all videos.

The overall gesture recognition rate of $99.76 \%$ is noted for private video sequences. To evaluate our system, we have developed a proof of concept app where users are asked to perform touch-less clicks and launch desired social media site (as shown in Figure 1). It demonstrates easy-to-use and accurate hand-free interactions.

\section{Conclusions}

Proposed approach allows user to perform touch-less interaction when their hand are covered and/or busy. We will conduct comprehensive user studies for checking validity and limitations of proposed interaction on smart phones. We believe that it's entirely possible to perform multi-modal tasks and communications using smart phone's touch-less interaction on go!

\section{Acknowledgement}

Authors would like to thank our colleagues Prof. Haibo Li at KTH and DML lab (Alaa Halawani, Shahrouz Yousefi, Farid Abedan Kondori) at Umea university for the fruitful discussions, and Prof. Shengzhong Feng for the kind help. Authors are thankful to the National Natural Science Fund of China (61070147).

\section{References}

[1] D. Wigdor, C. Forlines, P. Baudisch, J. Barnwell, and C. Shen. Lucid touch: a see-through mobile device, in Proc. 20th ACM Sym. UIST., pp. 269-278, 2007.

[2] S. Réhman, A. Khan, and H. Li, Interactive Feet for Mobile Immersive Interaction, in Proc. MobiVis at MobileHCI 2012.

[3] Z. Kalal, K. Mikolajczyk, and J. Matas. Tracking Learning Detection., IEEE Transactions on Pattern Analysis and Machine Intelligence, 6(1), 2010.

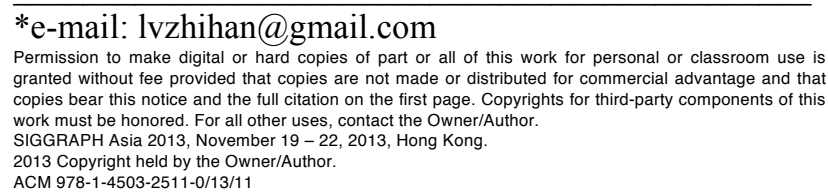

